\title{
Probing BFKL dynamics at hadronic colliders in jet gap jet events
}

\author{
Christophe Royon ${ }^{1, \star}$ Federico Deganutti ${ }^{1, \star \star}$ \\ ${ }^{1}$ Department of Physics and Astronomy, The University of Kansas, Lawrence, USA
}

\begin{abstract}
In this report, we give the Balitsky Fadin Kuraev Lipton formalism for jet gap jet events at hadronic colliders. We also discuss the case where in addition at least one proton is intact in the final state in diffractive events.
\end{abstract}

\section{Looking for BFKL resummation effects at hadronic colliders: forward jets and Mueller-Navelet jets}

The experimental search for resummation effects at low $x$ where $x$ is the momentum fraction of the interacting parton inside the proton, as predicted by the Balitsky Fadin Kuraev Lipatov (BFKL) [1] equation has been an important topic of research at recent colliders The starting point to look for BFKL resummation effects at colliders was to study forward jet production at HERA, the $e p$ collider located at DESY, Hamburg. When $Q$, the transferred energy between the electron and the interacting quark, is of the same order of the forward jet transverse momentum, and when the interval in rapidity between the forward jet and the scattered electron is large, the cross section predicted by usual Quantum Chromodynamics (QCD) as given by the Dokhitzer Gribov Lipatov Altarelli Parisi (DGLAP) [2] evolution equation is small because of the $k_{T}$-ordering of the different emitted gluons on the ladder. On the contrary, the BFKL dynamics predicts a non-negligible cross section. As an example, the $\mathrm{H} 1$ collaboration measured the triple differential forward jet cross section as a function of $Q^{2}$, jet $p_{T}$ and jet fractional energy [3]. The BFKL NLL formalism leads to a good description of data whereas the NLO QCD calculation has difficulties to describe the forward jet cross section [4]. However this measurement was not completely conclusive concerning the observation of low $x$ resummation effects since higher order QCD corrections lead to a good description of data.

The second observable that was proposed to look for BFKL resummation effects at hadronic colliders is the Mueller-Navelet jet production [5]. The idea is to look for jets separated will a large interval in rapidity. When the transverse momentum of the two jets is similar, the BFKL dynamics predicts a larger cross section than the DGLAP one because of the $k_{T}$ ordering of the different gluons along the ladder as before $[6,7]$. One possible observable is the measurement of dijet azimuthal decorrelation which is larger for BFKL dynamics because of the multiple gluon emission [6]. Unfortunately, this prediction suffers from high order corrections to BFKL dynamics that has a tendency to bring both predictions to be closer to each other. As an example, the most recent measurements by the

\footnotetext{
$\star$ e-mail: christophe.royon@ku.edu

$\star \star$ e-mail: fedeganutti@gmail.com
} 
CMS and ATLAS collaborations [8] are shown in Fig. 1. Data can be described by BFKL dynamics as expected but also by NLO DGLAP QCD calculations. It is thus needed to define new possible variables or going to larger interval of rapidity between jets, using the CASTOR [9] very forward calorimeter in CMS as an example, in order to enhance further the BFKL contribution.

New variables were recently proposed to look for BFKL dynamics [10]. The idea is to look for mini-jet production between the two Mueller-Navelet jets that are predicted by DGLAP or BFKL dynamics as illustrated in Fig. 2. The pattern for gluon emission is different between both dynamics because of the $k_{T}$-ordering of the gluons in the ladder for the DGLAP prediction. As an example, it would be possible to measure the mini-jet production with transerve momentum above 20 or $25 \mathrm{GeV}$ between the two Mueller Navelet jets with $p_{T}>40$ or $45 \mathrm{GeV}$.

In the next section, we will discuss another method to look for BFKL dynamics, namely the measurement of jet gap jet events.
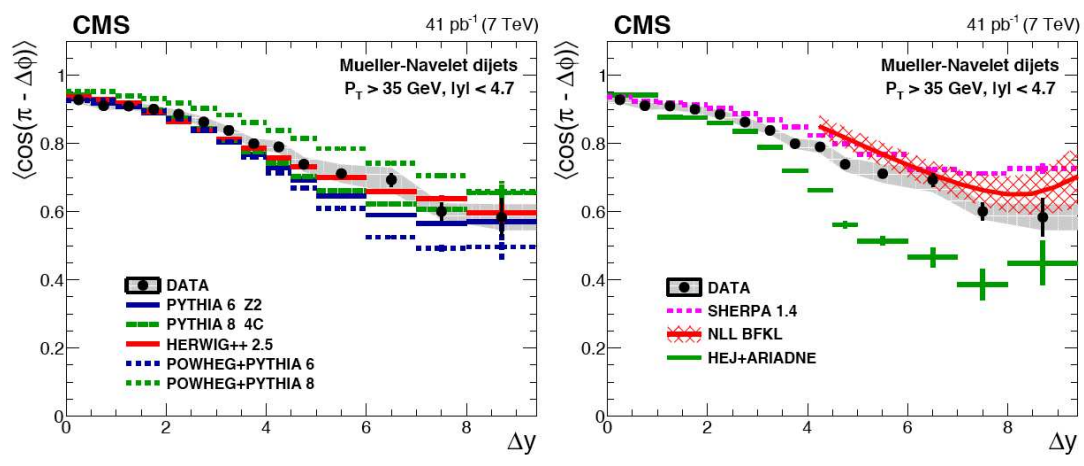

Figure 1. Measurement of the azimuthal decorrelation between Mueller Navelet jets measured by the CMS collaboration and compared to DGLAP Monte Carlo and BFKL calculations.

\section{Jet gap jet production}

\subsection{Gap between jets at the Tevatron and the LHC}

The measurement of gap between jets or the Mueller-Tang process [11] was performed at the Tevatron [12] and is also being done at the LHC [13]. The idea is to look for two jets separated by a large interval in rapidity $\Delta \eta$ with a gap devoid of any activity or energy (no particle emission) in a central part of the detector $\Delta \eta_{g a p}$ (for instance between (-1) and (1) in rapidity). This means that a colorless object, or a Pomeron, is emitted between the two jets. The natural description of this object is the BFKL Pomeron. The prediction of the BFKL cross section to produce these events is not negligible whereas the DGLAP prediction is very small for gap sizes above two units in rapidity. This is why this process is ideal to look for BFKL resummation effects. However, it suffers from potential additional interactions, such as soft gluon exchanges, that can destroy the gap between the jets. This is the so-called the survival probability that can be as low as 0.1 at Tevatron center-of-mass energy (about 2 $\mathrm{TeV}$ ) and 0.03 at the LHC (about $14 \mathrm{TeV}$ ). 


$$
\begin{gathered}
\left\langle p_{T}\right\rangle=\frac{1}{N} \sum_{i=1}^{N}\left|p_{T i}\right| \\
\left\langle R_{y}\right\rangle=\frac{1}{N+1} \sum_{i=1}^{N+1} \frac{y_{i}}{y_{i-1}}
\end{gathered}
$$
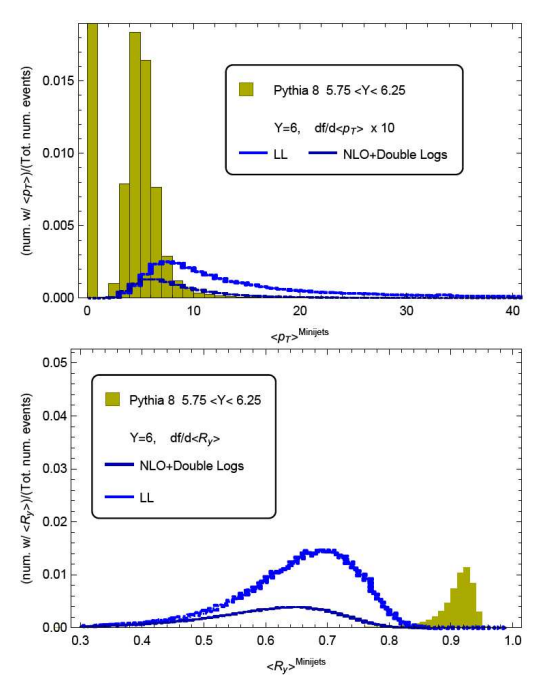

Figure 2. New less inclusive measurements in Mueller-Navelet jet events to look for BFKL resummation effects: average $p_{T}$ rapidity of mini-jet production.

\subsection{The NLL BFKL formalism and comparison with data}

In order to describe the measurements of gap between jets at the Tevatron and the LHC, the following BFKL formalism was used. The production cross section of two jets with a gap in rapidity between them reads

$$
\frac{d \sigma^{p p \rightarrow X J J Y}}{d x_{1} d x_{2} d E_{T}^{2}}=\mathcal{S} f_{e f f}\left(x_{1}, E_{T}^{2}\right) f_{e f f}\left(x_{2}, E_{T}^{2}\right) \frac{d \sigma^{g g \rightarrow g g}}{d E_{T}^{2}}
$$

where $\sqrt{s}$ is the total energy of the collision, $E_{T}$ the transverse momentum of the two jets, $x_{1}$ and $x_{2}$ their longitudinal fraction of momentum with respect to the incident hadrons, $S$ the survival probability, and $f$ the effective parton density functions. The rapidity gap between the two jets is $\Delta \eta=\ln \left(x_{1} x_{2} s / p_{T}^{2}\right)$.

The cross section is given by

$$
\frac{d \sigma^{g g \rightarrow g g}}{d E_{T}^{2}}=\frac{1}{16 \pi}\left|A\left(\Delta \eta, E_{T}^{2}\right)\right|^{2}
$$

in terms of the $g g \rightarrow g g$ scattering amplitude $A\left(\Delta \eta, p_{T}^{2}\right)$.

In the following, we consider the high energy limit in which the rapidity gap $\Delta \eta$ is assumed to be very large. The BFKL framework allows to compute the $g g \rightarrow g g$ amplitude in this regime, and the result is known up to NLL accuracy (in this calculation, the coupling to the proton, the so-called impact factors, was computed at LL)

$$
A\left(\Delta \eta, E_{T}^{2}\right)=\frac{16 N_{c} \pi \alpha_{s}^{2}}{C_{F} E_{T}^{2}} \sum_{p=-\infty}^{\infty} \int \frac{d \gamma}{2 i \pi} \frac{\left[p^{2}-(\gamma-1 / 2)^{2}\right]}{\left[(\gamma-1 / 2)^{2}-(p-1 / 2)^{2}\right]} \frac{\exp \left\{\bar{\alpha}\left(E_{T}^{2}\right) \chi_{e f f}\left[2 p, \gamma, \bar{\alpha}\left(E_{T}^{2}\right)\right] \Delta \eta\right\}}{\left[(\gamma-1 / 2)^{2}-(p+1 / 2)^{2}\right]}
$$

with the complex integral running along the imaginary axis from $1 / 2-i \infty$ to $1 / 2+i \infty$, and with only even conformal spins contributing to the sum, and $\bar{\alpha}=\alpha_{S} N_{C} / \pi$ the running coupling. 
In this study, we performed a parametrised distribution of $d \sigma^{g g \rightarrow g g} / d E_{T}^{2}$ so that it can be easily implemented in the HERWIG Monte Carlo [15] since performing the integral over $\gamma$ in particular would be too much time consuming in a Monte Carlo. The implementation of the BFKL cross section in a Monte Carlo is absolutely necessary to make a direct comparison with data. Namely, the measurements are sensitive to the jet size (for instance, experimentally the gap size is different from the rapidity interval between the jets which is not the case by definition in the analytic calculation).

Let us first notice that the sum over all conformal spins is absolutely necessary. Considering only $p=0$ in the sum of Equation 3 leads to a wrong normalization and a wrong jet $E_{T}$ dependence, and the effect is more pronounced as $\Delta \eta$ diminishes.

The DØ Collaboration measured the jet gap jet cross section ratio with respect to the total dijet cross section, requesting for a gap between -1 and 1 in rapidity, as a function of the second leading jet $E_{T}$, and $\Delta \eta$ between the two leading jets for two different low and high $E_{T}$ samples $\left(15<E_{T}<20\right.$ $\mathrm{GeV}$ and $E_{T}>30 \mathrm{GeV}$ ). To compare with theory, we compute the following quantity

$$
\text { Ratio }=\frac{\text { NLL HERWIG }}{\text { LO Herwig }} \times \frac{\text { LO QCD dijets }}{\text { NLO QCD dijets }}
$$

in order to take into account the NLO corrections on the dijet cross sections, where BFKL NLL HERWIG and Dijet HERWIG denote the BFKL NLL and the dijet cross section implemented in HERWIG [15]. The NLO QCD cross section was computed using the NLOJet++ program [16].

The comparison with DØ data [12] is shown in Fig. 3. We find a good agreement between the data and the BFKL calculation. It is worth noticing that the BFKL NLL calculation leads to a better result than the BFKL LL one (note that most studies in the literature considered only the $p=0$ component which is not a valid assumption). The comparison with CDF data [12] leads to a similar conclusion and data are well described by the BFKL NLL formalism.

The latest results have been provided by the CMS Collaboration using data collected in pp collisions at $\sqrt{s}=7 \mathrm{TeV}$ [13]. Each of the leading two jets has transverse momentum of $p_{\mathrm{T}}>40 \mathrm{GeV}$, pseudorapidity of $1.4<\left|\eta_{\text {jet }}\right|<4.7$, and opposite signed pseudorapidities $\eta_{\text {jet } 1} \cdot \eta_{\text {jet } 2}<0$. The fraction of jet-gap-jet events were measured as a function of $p_{\mathrm{T}}$, jet 2 and pseudorapidity difference between the leading two jets $\Delta \eta_{\mathrm{jj}}$.

\subsection{The NLL BFKL formalism including NLO impact factors}

The full NLL BFKL calculation using the NLO impact factor is in progress [18]. Several complications appear when the impact factors are refined to NLO.

- The back-to-back symmetry proper to an elastic interaction is lost since a third parton can appear in the final state.

- Since the NLO impact factor interference diagrams connect in a non trivial way to the gluon-Green function (GGF) their convolution cannot be factorized any more into an amplitude square involving only the integrated GGF. Moreover, the GGF in its full form is written in terms of Gausshypergeometric functions in some particularly complicated combinations especially for large conformal spins and it complicates the numerical implementation.

- A formal divergence appears in the real-corrections published in [19] when the proper jet reconstruction algorithm is employed. The problematic behavior appears when besides the two tagged jets, a third parton is emitted in the gap region but with a sub-threshold transverse energy. One would think that such configuration should be suppressed when the additional parton becomes more central. This puzzling behavior motivates an independent cross-check of previously pubished results that is currently a work-in-progress. 

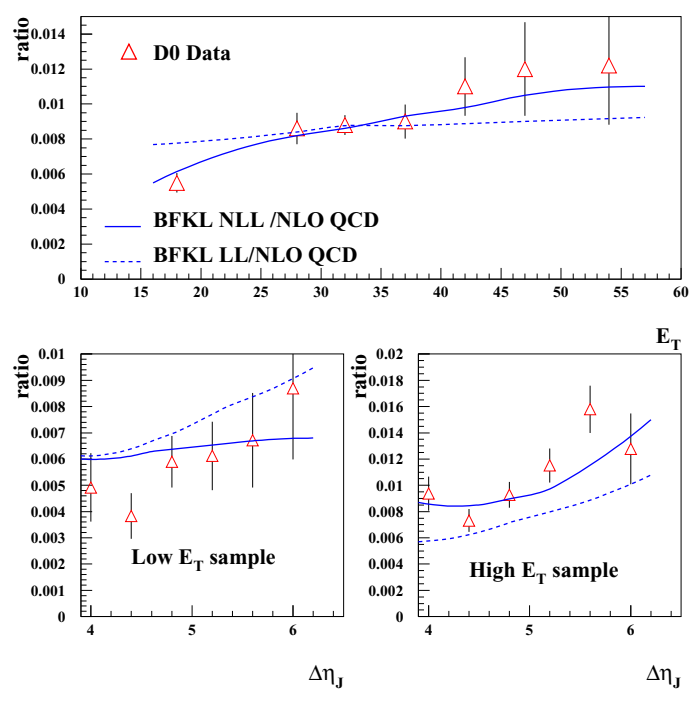

Figure 3. Comparison between the measurement of gap between jets events by the DO collaboration and the BFKL calculation as a function of jet $E_{T}$ or $\Delta \eta$ between jets for the low and high $E_{T}$ samples.

It is expected that the full NLO calculation will be available soon, leading to more accurate comparison with data.

\subsection{Jet gap jet events in diffraction}

In this section, we discuss the observation of gap between jets in single diffractive or double Pomeron exchange events [20]. These processes are similar to the one discussed in the previous section but they require in addition the existence of at least one intact proton after interaction. This process was implemented in the FPMC generator [17].

The DPE jet-gap-jet event ratio is defined as the ratio of the cross section for the DPE jet-gap-jet (JGJ) production to the DPE inclusive dijet (Jets) production $R=\sigma(D P E \mathrm{JGJ}) / \sigma(D P E$ Jets) and is plotted in Fig. 4 as a function of the transverse momenta of the first-leading jet. To take into account NLO QCD effects, absent in the FPMC program, the LO ratio obtained was corrected by the cross section ratio $\sigma(D P E L O J e t++) / \sigma(D P E N L O$ Jet ++$)$, obtained by the NLO Jet++ program [16].

As far as the gap fraction $\sigma(D P E J G J) / \sigma(D P E$ Jets $)$ is concerned, note that we did not consider an additional suppression factor for DPE jet-gap-jet production, on top of the 0.03 of DPE inclusive jet production. Therefore, in the predictions of Fig. 4, all the rapidity gap survival probability cancel. We would like to point out that this last point is an assumption, DPE jet-gap-jet production could be subject to a bigger suppression than DPE inclusive jet production, due to the extra color-singlet exchange in the hard cross section. However, we do not expect this potential additional factor (on top of 0.03 ) to be large, due to the fact that extra soft interactions with the BFKL pomeron are unlikely: the 2-to-2 hard scattering takes place on much shorter time scale compared to the soft interactions filling the rapidity gaps. In any case, this will be checked at the LHC and if necessary, our numbers can then be adjusted accordingly. 


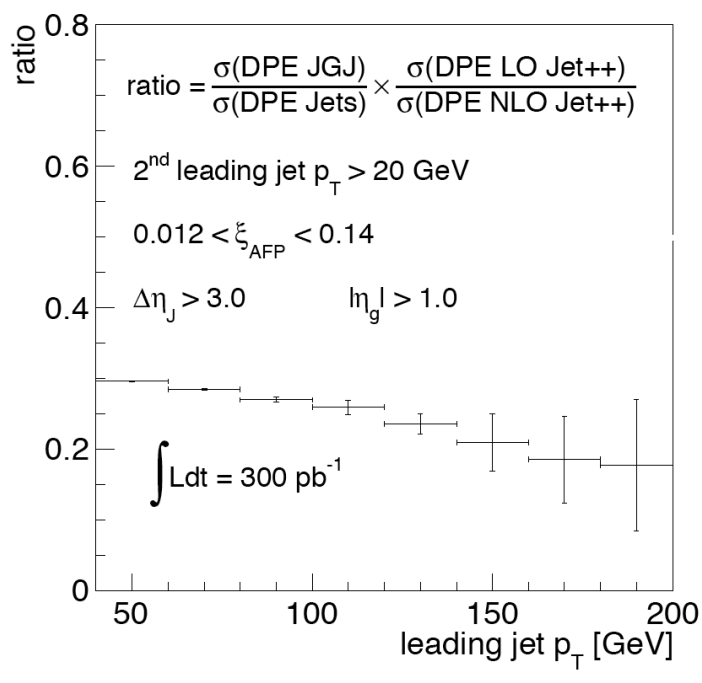

Figure 4. Ratio of the jet-gap-jet to the inclusive jet cross sections at the LHC as a function of jet $p_{T}$ in double pomeron exchange events where the protons are detected in AFP or CT-PPS.

\section{Conclusion}

\section{References}

[1] V. S. Fadin, E. A. Kuraev, L. N. Lipatov, Phys. Lett. B60 (1975) 50; L. N. Lipatov, Sov. J. Nucl. Phys. 23 (1976) 338; E. A. Kuraev, L. N. Lipatov and V. S. Fadin, Sov. Phys. JETP 45 (1977) 199;I. I. Balitsky, L. N. Lipatov, Sov.J.Nucl.Phys. 28 (1978) 822; V.S. Fadin and L.N. Lipatov, Phys. Lett. B429 (1998) 127; M. Ciafaloni, Phys. Lett. B429 (1998) 363; M. Ciafaloni and G. Camici, Phys. Lett. B430 (1998) 349.

[2] G.Altarelli and G.Parisi, Nucl. Phys. B126 18C (1977) 298; V.N.Gribov and L.N.Lipatov, Sov. Journ. Nucl. Phys. (1972) 438 and 675; Yu.L.Dokshitzer, Sov. Phys. JETP. 46 (1977) 641.

[3] H1 Coll., Eur. Phys. J. C46 (2006) 27; ZEUS Coll., Phys. Lett. B 632 (2006) 13.

[4] O. Kepka, C. Royon, C. Marquet, R. Peschanski, Eur. Phys. J. C55 (2008) 259; O. Kepka, C. Royon, C. Marquet, R. Peschanski, Phys. Lett. B655 (2007) 236; O. Kepka, C. Royon, C. Marquet, R. Peschanski, Eur. Phys. J. C55 (2008) 259; C. Marquet, C. Royon, Nucl. Phys. B739 (2006); H. Navelet, R. Peschanski, C. Royon, S. Wallon, Phys. Lett. B385 (1996) 357; H. Navelet, R. Peschanski, C. Royon, Phys. Lett. B366 (1996) 329; J.G. Contreras, R. Peschanski and C. Royon, Phys. Rev. D62 (2000) 034006; C. Marquet, R. Peschanski and C. Royon, Phys. Lett. B599 (2004) 236.

[5] A.H. Mueller, H. Navelet, Nucl. Phys. B282 (1987) 727.

[6] C. Marquet. C. Royon, Phys. Rev. D79 (2009) 034028.

[7] B. Ducloué, L. Szymanowski, S. Wallon, JHEP 1305 (2013) 096; D. Colferai, F. Schwennsen, L. Szymanowski, S. Wallon, JHEP 1012 (2010) 026; F. Caporale, F.G. Celiberto, G. Chachamis, D. Gordo Gomez, A. Sabio Vera, Phys. Rev. D95 (2017) no.7, 074007; M. Angioni, G. Chachamis, J.D. Madrigal, A. Sabio Vera, Phys. Rev. Lett. 107 (2011) 191601; A. Sabio Vera and F. 
Schwennsen, Nucl. Phys. B 776 (2007) 170; A. Sabio Vera and F. Schwennsen, Phys. Rev. D77 (2008) 014001; B. Ducloue, L. Szymanowski, S. Wallon, Phys. Rev. Lett. 112 (2014) 082003.

[8] CMS Coll., JHEP 08 (2016) 139; ATLAS Coll. Eur. Phys. J. C74 (2014) 3117.

[9] CMS Coll. Eur. Phys. J. C67 (2010) 601-615.

[10] G. Chachamis, A. Sabio Vera, JHEP 1602 (2016) 064; G. Chachamis, A. Sabio Vera, Phys.Rev. D93 (2016) no.7, 074004; C. Baldenegro, F. Deganutti, D. Gordo, M. Klasen, C. Royon, J. Salomon, in preparation

[11] A. H. Mueller, W. K. Tang, Phys. Lett. B 284 (1992) 123.

[12] B. Abbott et al., Phys. Lett. B 440, 189 (1998); F. Abe et al., Phys. Rev. Lett. 80, 1156 (1998).

[13] CMS Coll., Eur, Phys. J. C78 (2018) 242.

[14] O. Kepka, C. Marquet, C. Royon, Phys. Rev. D83 (2011) 034036; F. Chevallier, O. Kepka, C. Marquet, C. Royon, Phys. Rev. D79 (2009) 094019.

[15] G. Marchesini et al., Comp. Phys. Comm. 67, 465 (1992).

[16] Z. Nagy and Z. Trocsanyi, Phys. Rev. Lett. 87, 082001 (2001).

[17] M. Boonekamp, A. Dechambre, V. Juranek, O. Kepka, M. Rangel, C. Royon, R. Staszewski, ePrint: arXiv:1102.2531; M. Boonekamp, V. Juranek, O. Kepka, C. Royon "Forward Physics Monte Carlo", "Proceedings of the workshop: HERA and the LHC workshop series on the implications of HERA for LHC physics," arXiv:0903.3861 [hep-ph].

[18] D. Colferai, F. Deganutti, C. Royon, T, Raben, in preparation.

[19] M. Hentschinski, J. D. Madrigal Martinez, B. Murdaca, A. Sabio Vera, Nucl. Phys. B887 (2014) 309.

[20] C. Marquet, C. Royon, M. Trzebinski, R. Zlebcik, Phys.Rev. D87 (2013) 3, 034010; O. Kepka, C. Marquet, C. Royon, Phys. Rev. D83 (2011) 034036. 